\title{
Ventilation through laryngeal mask in patient with severe laryngeal stenosis
}

\author{
Tsvetomir Marinov, MD, PhD \\ Department of Anesthesiology and Intensive Care \\ University Hospital "Queen Giovanna" - ISUL \\ Medical University - Sofia
}

\begin{abstract}
Introduction: Patients with an advanced airway obstruction and inspiratory stridor at rest comprise some of the most feared and complicated cases for the anesthesiologist.

Case description: A 45-year-old female patient (weight $54 \mathrm{~kg}$, height $166 \mathrm{~cm}$ ), ASA III, was admitted to our hospital for tracheostomy and microlaryngeal surgery because of stenosis of the larynx. A general anesthesia was scheduled for this patient. Fentanyl $0.3 \mathrm{mg}$, propofol $200 \mathrm{mg}$ and succinylcholine $100 \mathrm{mg}$ were administered for anesthesia induction. After intubation $2.5 \%$ sevoflurane was used for anesthesia maintenance. Additional $0.15 \mathrm{mg}$ of fentanyl was administered for pain relief. Muscle relaxation was provided with $40 \mathrm{mg}$ of atracurium besilate. The operation finished successfully within $80 \mathrm{~min}$. Ventilation for tracheostomy was successfully performed through flexible laryngeal mask airway (FLMA) № 3.

Discussion: Patients presenting for microlaryngeal surgery frequently have a difficult airway. The chosen approach to this airway depends on the pathology and the patient's symptoms. Acceptance of the LMA as a safe practical alternative to tracheal intubation for laryngeal stenosis requires that placement be relatively facile, that position of the mask be stable throughout surgery, and that the airway be protected from the risk of aspiration. In our study during maintenance of anesthesia, there was no evidence of instability of the LMA or increased contamination of the lower respiratory tract.

Conclusion: Ventilation through LMA can be safely used during tracheostomy in patients with severe laryngeal stenosis.

Key words: laryngeal stenosis, laryngeal mask, ventilation
\end{abstract}

\section{Introduction}

Patients with an advanced airway obstruction and inspiratory stridor at rest comprise some of the most feared and complicated cases for the anesthesiologist. The incidence of difficult mask ventilation and impossible mask ventilation among patients with severe stridor and upper airway obstruction of more than $75 \%$ of the lumen reaches $40 \%$ and $6 \%$, respectively, ${ }^{1}$ compared with $1.4 \%$ and $0.15 \%$ for the general surgical population ${ }^{2}$. These patients frequently present for microlaryngeal surgery on an emergent or semi-emergent basis, yet they require a systematic and thoughtful approach by the anesthesiologist and the surgeon. The nature of the obstructing lesion (e.g., vascular, submucosal, pedunculated, inflammatory) and its location (e.g., supraglottic, glottic, subglottic, midtracheal, lower tracheal, and bronchial [mediastinal]) may require completely different intubation considerations and approaches.

In the context of laryngeal surgery, the optimal technique of airway management of the stridorous patient with an advanced proximal airway obstruction (i.e., supraglottic, glottic, and subglottic levels) remains a subject of controversy. An awake flexible fiberoptic intubation, inhalational induction, and intravenous induction with muscle relaxants have been used successfully, but none should be considered fail-safe ${ }^{3}$. Thorough preoperative discussion of the surgical pathology and formulation of closely coordinated airway management plan with the 
surgeon are essential for safe management of these patients.

The laryngeal mask airway (LMA) has become an important adjunct to airway management since its introduction in $1988^{4}$. It provides more hands-free anesthesia than a facemask does, avoids many morbidities associated with tracheal intubation because there is no stress from the laryngoscope, and allows a faster recovery that does not require muscle relaxation ${ }^{5}$. Various types of LMAs have been developed. The flexible laryngeal mask airway (FLMA) was first used successfully in tonsillectomies and dental surgeries in 1990 to prevent the obstruction and kinking observed when using classical LMA tubes. Since then, the FLMA has been used in thyroidectomies, cleft palate surgeries, nasal surgeries, head and neck oncologic surgeries ${ }^{6}$. There is a general tendency towards using supraglottic devices in ear, nose and throat (ENT) surgeries. Unlike many surgeries, there is no need for high intraoperative inspiratory pressure. Although the patient's head is out of reach, it is usually fixed during the whole length of the surgery. Laryngeal mask can effectively protect lower airways against blood, secretions and irrigation fluids during surgery. Even in prolonged ENT surgeries, supraglottic devices are good choices and the length of surgery is not a definite limiting factor for their use ${ }^{7}$.

\section{Case report}

It is reported in the case airway instrumentation through laryngeal mask during tracheostomy in patient with severe laryngeal obstruction.

A 45-year-old female patient (weight $54 \mathrm{~kg}$, height $166 \mathrm{~cm}$ ), ASA III, was admitted to our hospital for tracheostomy and microlaryngeal surgery because of stenosis of the larynx. A general anesthesia was scheduled for this patient. Fentanyl $0.3 \mathrm{mg}$, propofol $200 \mathrm{mg}$ and succinylcholine $100 \mathrm{mg}$ were administered for anesthesia induction. After intubation $2.5 \%$ sevoflurane was used for anesthesia maintenance. Additional $0.15 \mathrm{mg}$ of fentanyl was administered for pain relief. Muscle relaxation was provided with $40 \mathrm{mg}$ of atracurium besilate. The operation finished successfully within $80 \mathrm{~min}$. The vital signs had been stable during the operation.

Ventilation during tracheostomy was successfully performed through flexible laryngeal mask airway (FLMA) № 3. The FLMA was advanced by pressure against the hard palate to follow the oropharyngeal curve, advancing its tube with the other hand and oropharyngeal leak around FLMA was detected by noise during manual bag ventilation. Mechanical ventilation was started using a ventilator (Datex Ohmeda Aespire) in the volume control mode. A tidal volume of $550 \mathrm{ml}$, a respiratory rate of 11 and an inspiratory: expiratory ratio of 1:2 was used. Normocarbia was maintained (end tidal carbon dioxide 34-38 $\mathrm{mmHg}$ ). Adequate ventilation was judged by end tidal carbon dioxide $\left(\mathrm{ETCO}_{2}\right)$ and expired tidal volume. Standard intraoperative monitoring was applied (non invasive blood pressure at 5 minutes interval, ECG, pulse oximetry and capnography). During maintenance of anesthesia, there was no evidence of instability of the FLMA, compromising of ventilation or contamination of the lower respiratory tract. After tracheostomy was finished FLMA was removed and patient was intubated with ETT №7 through tracheostomy foramen (fig.1).

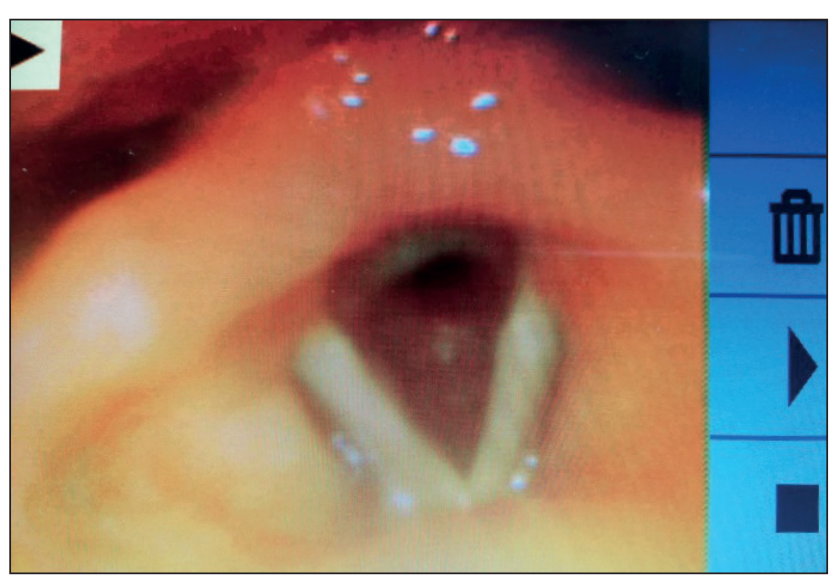

Fig.1. Severe laryngeal stenosis

Extubation was performed when the patient restores reflexes and spontaneous breathing with tidal volume of $5 \mathrm{ml} / \mathrm{kg}$. Discharge criteria from the operating room were normal respiratory pattern, adequate tidal volume and $\mathrm{SpO}_{2} \geq 98 \%$, and appropriate response to verbal stimuli, then patient was transferred to post anesthetic care unit (fig.2).

\section{Discussion}

Patients presenting for microlaryngeal surgery frequently have a difficult airway. The chosen approach to this airway depends on the pathology and the patient's symptoms. In situations with critical airway compromise, an awake tracheostomy may be warranted from the outset, but it may prove to be technically challenging or impossible and may require general anesthesia. Even if an awake 

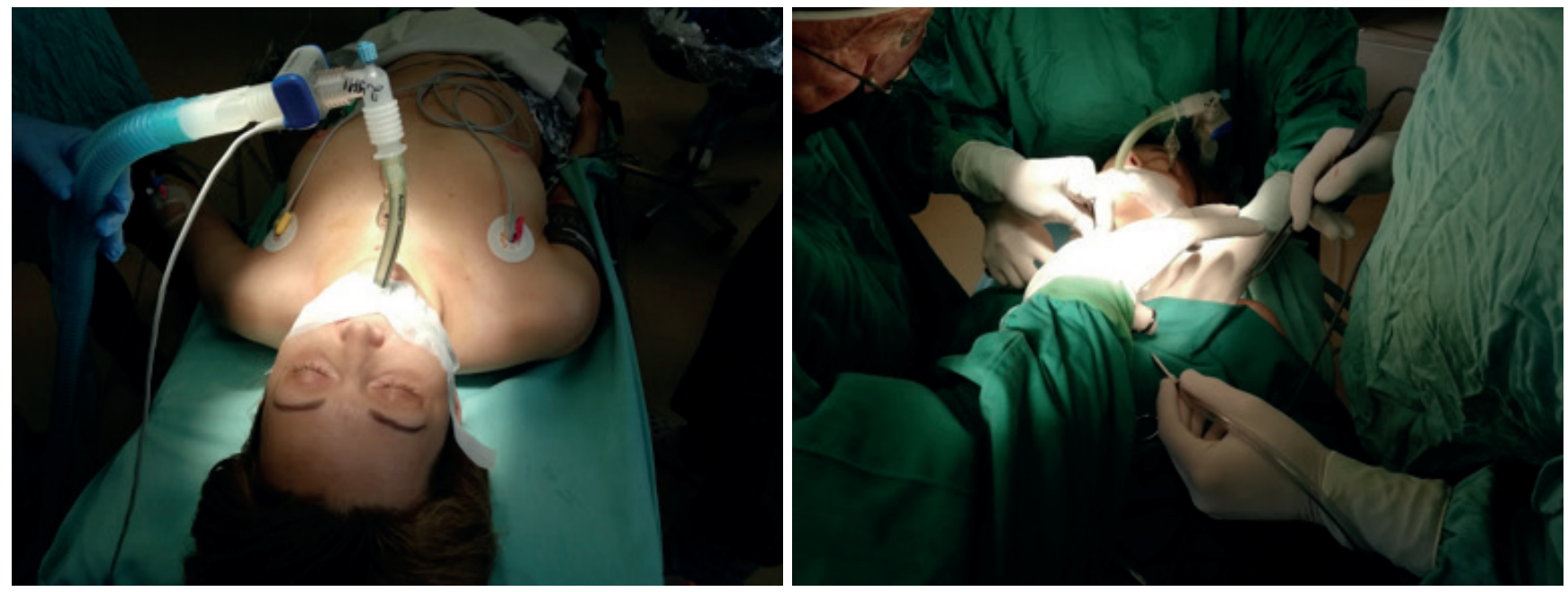

Fig.2. Ventilation through laryngeal mask in patient with severe laryngeal stenosis

tracheostomy is chosen as a primary approach, full backup preparation for alternative airway management is necessary. Video laryngoscopy reliably improves laryngeal exposure by at least one grade, allows continuous observation of the entire intubation procedure by the entire team, and may therefore be a near-ideal technique for managing difficult airways in patients presenting for microlaryngeal surgery. Choosing the video laryngoscopic device depends on the operator's preference and must consider the nature and location of the lesions. The decision to proceed with an awake or asleep approach to an anticipated difficult airway should follow the American Society of Anesthesiologists (ASA) difficult airway algorithm, with special attention directed to predictors of difficult mask ventilation, impossible mask ventilation, and their association with difficult intubation ${ }^{8}$. The anesthesiologist also should review the pertinent preoperative findings identified on flexible fiberoptic laryngoscopy, chest radiography, CT, and MRI and should discuss these findings with the surgeon.

Acceptance of the LMA as a safe practical alternative to tracheal intubation for laryngeal stenosis requires that placement be relatively facile, that position of the mask be stable throughout surgery, and that the airway be protected from the risk of aspiration. Because the larynx is not directly stimulated, respiratory and cardiovascular reflex responses to placement and removall of the LMA are reduced compared with those after tracheal intubation.

In our study during maintenance of anesthesia, there was no evidence of instability of the LMA or increased contamination of the lower respiratory tract.

Stenosis in the area of the cricotracheal segment is still a challenging problem to be dealt with. Post-intubational cases mark an increase in recent years due to the advances in intensive care, thoracic surgery and neurosurgery departments. Popov et al. describe a case report of a patient with severe subglottic stenosis. A 35-year old male patient was admitted to the Department of Otorhinolaryngology with a compensated inspiratory dyspnea and biphasic stridor. Video fibroscopy revealed a severe subglottic stenosis grade III according to the Cotton-Myer scale. Computed tomography scan of the neck and chest confirmed an hourglass-shaped subglottic stenosis which carried down to the second tracheal ring. General anesthesia was induced and patient was ventilated successfully through a LMA ${ }^{9,10}$.

\section{Conclusion}

The LMA has become an important adjunct to airway management since its introduction. Ventilation through FLMA can be safely used during tracheostomy in patients with severe laryngeal stenosis. 


\section{References}

1. Ross-Anderson DJ, Ferguson C, Patel A: Transtracheal jet ventila $\neg$ tion in 50 patients with severe airway compromise and stridor. Br J Anaesth 106:140-144, 2011.

2. Langeron O, Masso E, Huraux C, et al: Prediction of difficult mask ventilation. Anesthesiology 92:1229-1236, 2000.

3. Moorthy SS, Gupta S, Laurent B, Weisberger EC: Management of airway in patients with laryngeal tumors. J Clin Anesth 17:604-609, 2005.

4. Maltby JR, Loken RG, Watson NC. The laryngeal mask airway: clinical appraisal in 250 patients. Can J Anaesth 1990;37:509 -13.

5. Hernandez MR, Klock PA Jr., Ovassapian A. Evolution of the extraglottic airway: a review of its history, applications, and practical tips for success. Anesth Analg. 2012; 114: 349-368.

6. Alexander CA. A modified Intavent laryngeal mask for ENT and dental anaesthesia. Anaesthesia. 1990; 45: 892-893.

7. Thornton JA. Anesthesia for Eye, Ear, Nose, and Throat Surgery. Br Med J. 2010;3:547.

8. American Society of Anesthesiologists Task Force on Management of the Difficult Airway: Practice guidelines for management of the difficult airway: An updated report by the American Society of Anesthesiologists Task Force on Management of the Difficult Airway. Anesthesiology 98:1269-1277, 2003.

9. Sapundzhiev, N., Kiryazov, V., Korucheva, K., Tsonev, A., Platikanov, V. Postintubational laryngotracheal stenosis. Analysis of two cases. Causes and prevention. Anaesthesiology and Intensive Care, 2007, 34(5), pp. 7-10.

10. Popov, T. M., Marinov, T., Rangachev, J., Konov, D., \& Belitova, M. (2017). Cricotracheal resection in a patient with severe subglottic stenosis-advantages of a temporary non-cannulated tracheostomy. Balkan medical journal, 34(2), 165.

Reviewers: Assoc. prof. Dr. Alexander Vulkov Vulkov / Prof. Dr. V. Platikanov

\section{Correspondence to:}

Tsvetomir Marinov, MD, PhD

Department of Anesthesiology and Intensive Care

University Hospital "Queen Giovanna"- ISUL

Byalo More Str 8

Medical University - Sofia

E-mail:ts.marinov@abv.bg 